BRX-TH 533

\title{
A Note on Stress-Tensors, Conservation and Equations of Motion
}

\author{
S. Deser \\ Department of Physics, Brandeis University \\ Waltham, MA 02454, USA
}

(November 20, 2016)

\begin{abstract}
Some unusual relations between stress tensors, conservation and equations of motion are briefly reviewed.
\end{abstract}

Asher Peres is a master of the subtle and unexpected, particularly in general relativity, his first love. I therefore present him with this short collection of (slightly) unlikely relations, from both sides of the Einstein equations, involving stress tensors, conservation and equations of motion.

Consider first point systems, whose stress tensors (and all other properties) are entirely localized along a world line; their generic form is then

$$
T^{\mu \nu}(x)=\int d s\left[a^{\mu}(s) \frac{d x^{\nu}}{d s}+N^{\mu \nu}(s)\right] \delta^{(m)}(x-x(s)), \quad N^{\mu \nu} \frac{d x_{\nu}}{d s}=0 .
$$

This is truly a minimal definition in terms of the a priori undefined vector and tensor functions $\left(a^{\mu}, N^{\mu \nu}\right)$ on the world line in dimension $m$. The "surprise", established in relatively recent times [1], is that once we demand conservation, $\partial_{\mu} T^{\mu \nu}=0$, this requirement alone fixes the stress tensor to be that of a free point particle:

$$
a^{\mu}=d x^{\mu} / d s, d a^{\mu} / d s=0, \quad N^{\mu \nu}(s)=0 .
$$

That is, conservation alone dictates the $T^{\mu \nu}$ to be just that of a freely moving point particle. The existence of an action can then be inferred uniquely from these "constraints", to which it is entirely equivalent. [As an aside, we mention that demanding conservation of a similarly localized vector current $j^{\mu}(x)=\int d s v^{\mu}(s) \delta^{(m)}(x-x(s))$ yields, as it should, the weaker result of continuity of the world line described by $v^{\mu}$, that is, particle conservation.] These results can be extended to a curved background, by demanding covariant conservation there.

The next surprise is that the same reasoning extends to strings $[2,3]$ : given a generic $T^{\mu \nu}$ localized on a 2-dimensional world sheet,

$$
T^{\mu \nu}(x)=\int d \tau^{1} d \tau^{2} \tau^{\mu \nu}(\tau) \delta^{(m)}\left(x-x\left(\tau^{a}\right)\right),
$$

conservation forces $\tau^{\mu \nu}$ to be that of a string,

$$
\tau^{\mu \nu}\left(\tau^{\mu}\right)=\sqrt{g} g^{a b} \frac{\partial x^{\mu}}{\partial \tau^{a}} \frac{\partial x^{\nu}}{\partial \tau^{b}},
$$


where $g_{a b}$ is the intrinsic world-sheet metric. Again conservation has fully determined both content and dynamics of the system. The converse surprise [2] is that higher dimensional, but still localized, higher membranes do not share this property of being fully determined by $T^{\mu \nu}$ conservation.

Can one extend these considerations to fields? With localization lost, much more information is required. For example, if we specify the stress tensor for a scalar field, say,

$$
T_{\mu \nu}=\phi_{\mu} \phi_{\nu}-\frac{1}{2} \eta_{\mu \nu} \phi_{\alpha} \phi^{\alpha}, \quad \phi_{\mu} \equiv \partial_{\mu} \phi
$$

then $\partial_{\mu} T^{\mu \nu}=\phi_{\nu} \square \phi$ clearly requires $\square \phi=0$ (which includes $\phi_{\nu}=0$ ). On the other hand, given the form of $T_{\mu \nu}$ in (5), we know it is uniquely equivalent to the metric variation of the normal scalar action (evaluated at flat space, for simplicity). From this it follows that $T^{\mu \nu}$ is conserved on- and only on-shell, since the action

$$
I=-\frac{1}{2} \int(d x) \sqrt{-g} g^{\mu \nu} \phi_{\mu} \phi_{\nu},
$$

is diffeomorphism invariant under arbitrary gauge functions $\xi_{\mu}(x)$; hence

$$
\delta I=0=\int\left(\xi_{\mu}(x) D_{\nu} T^{\mu \nu}+\int\left(\delta_{\xi} \phi\right) \frac{\delta L}{\delta \phi}\right.
$$

and the last term is the field variation contribution. Effectively then, given a form for $T^{\mu \nu}$, we know it is only of interest if derivable from an action, in which case we are in the usual circumstances of field theory. [If we had instead chosen a "bad" $T_{\mu \nu}$, say $T_{\mu \nu}=\phi_{\mu} \phi_{\nu}$, then $\partial_{\mu} T_{\nu}^{\mu}=\phi_{\nu} \square \phi+\frac{1}{2} \partial_{\nu} \phi_{\alpha}^{2}$, whose vanishing leaves no interesting dynamics.]

There is an additional sublety, depending on whether the action is in first or second order form, as we illustrate (for a change) through the vector field. If we assume the usual form of $T_{\mu \nu}$,

$$
T_{\mu \nu}=F_{\mu \alpha} F_{\nu}^{\alpha}-\frac{1}{4} g_{\mu \nu} F_{\alpha \beta}^{2},
$$

and that $F_{\mu \nu}$ is a curl, then conservation of $T_{\mu \nu}$ of course reduces to the requirement

$$
F_{\mu \alpha} \partial_{\beta} F^{\beta \alpha}=0 \text {. }
$$

Since (7) is also the metric variation of the (second-order) Maxwell action,

$$
I_{M}=-\frac{1}{4} \int \sqrt{g} g^{\mu \alpha} g^{\nu \beta} F_{\mu \nu} F_{\alpha \beta}
$$

this simply parallels the scalar discussion. However, we also know that there is a first order form of $I_{M}$, with $\left(A_{\mu}, F^{\mu \nu}\right)$ independent,

$$
I_{M}[A, F]=-\frac{1}{2} \int(d x)\left[F^{\mu \nu}\left(\partial_{\mu} A_{\nu}-\partial_{\nu} A_{\mu}\right)-\frac{1}{2} F^{\mu \alpha} F^{\nu \beta} g_{\mu \nu} g_{\alpha \nu} \sqrt{-g}\right] .
$$

In this form, both $\partial_{\mu} F^{\mu \nu}=0$ and $F_{\mu \nu}=\left(\partial_{\mu} A_{\nu}-\partial_{\nu} A_{\mu}\right)$ are on a par as field equations, and the symplectic $F \partial A$ term does not contribute to the metric variation ( $F^{\mu \nu}$ is here a contravariant density). The Maxwell tensor form (7) remains, however, but its conservation now requires that both these field equations hold, since $F^{\mu \nu}$ in (7) is now just an algebraic variable.

Returning to the condition (9) in second order form, obtaining the Maxwell equation from it requires proving that it implies $\partial_{\beta} F^{\beta \mu}=0$, i.e., that the determinant of its coefficient, $\operatorname{det}\left|F_{\alpha \beta}\right|$, vanish. Now this determinant is of the generic form $\alpha\left(E^{2}-B^{2}\right)^{2}+\beta(E \cdot B)^{2}$, and vanishes for 
plane waves, $E \cdot B=0,|E|=|B|$. So generically, stress tensor conservation implies field equations (which plane waves also obey) for the Maxwell system too.

More generally, one might draw the usual conclusion that for any matter system describable by a diffeo-invariant action, hence endowed with a well-defined $T^{\mu \nu}$ (up to the usual identically conserved superpotential terms), its conservation is generically equivalent to satisfaction of the corresponding field equations. But, there is yet another twist on the stress tensor-action connection. Two inequivalent actions can share the same $T^{\mu \nu}$ ! One example is any term, such as the ChernSimons (CS) invariant, whose action is topological i.e., metric-independent. Hence it does not contribute to $T^{\mu \nu}$ at all, but it had better - and does - preserve conservation; how? The simplest example is $D=3$ vector gauge theory with CS term; here is the abelian version:

$$
I_{T M E}=-\frac{1}{4} \int d^{3} x F_{\mu \nu}^{2}+m / 2 \int d^{3} x \epsilon^{\mu \nu \alpha} A_{\mu} F_{\nu \alpha} .
$$

Conservation of the normal Maxwell tensor (8) is "saved" by an identity. The field equation $\partial_{\mu} F^{\mu \nu}+m \epsilon^{\nu \alpha \beta} F_{\alpha \beta}=0$ implies that

$$
F_{\nu \alpha} \partial_{\beta} F^{\beta \alpha}=-F_{\nu \alpha} m \epsilon^{\alpha \lambda \sigma} F_{\lambda \sigma} \equiv 0 .
$$

Other such "topological" terms include, in $D=4$, the truncated Born-Infeld action $\int\left(d^{4} x\right) \sqrt{\operatorname{det} F_{\mu \nu}}$, which is also clearly metric-independent. In this sense, stress tensor conservation need not fully determine the action it represents - just the opposite of our initial localized examples.

Our final case is the gravitational field itself, which of course has no well-defined stress tensor. To what extent, then, can we construct parallel results to those valid for matter? Clearly, any geometric scalar density Lagrangian

$$
I=\int(d x) \sqrt{-g} L\left(R, R^{2},(D R) \ldots\right),
$$

has identically diffeo-invariant action, implying the (generalized) Bianchi identity for its metric variation, $\delta I / \delta g_{\mu \nu}$. But we are not interested in identically conserved quantities. This is how the only other tensor available, that of Bel-Robinson, entered Einstein gravity. We recall in $D=4$,

$$
T_{\alpha \beta \gamma \delta}=R_{\alpha \rho \gamma \sigma} R_{\beta}{ }_{\delta}^{\rho \sigma}+{ }^{*} R_{\alpha \rho \gamma \sigma}{ }^{*} R_{\beta}{ }_{\delta}^{\rho \sigma}
$$

is (covariantly) conserved by virtue of Ricci flatness; indeed that was its original appeal. In its cleanest form, with the curvature in (14) replaced by Weyl tensors, conservation clearly depends only on derivatives (say curls) of the Ricci tensor, rather than on $R_{\mu \nu}$ or $G_{\mu \nu}$ algebraically. Then [even apart from the plane-wave determinant ambiguities akin to the Maxwell case from $\sim R(D R)=0$ ] this means that weaker field equations, namely those where $R_{\mu[\alpha ; \beta]}=0$ also conserve the same $T_{\mu \nu \alpha \beta}$. This is amusing since these latter field equations are not derivable as a Lagrangian system, though they admit Einstein spaces.

There is however, something a bit strange here compared to lower spins. Since $B R$ is a 4 index quantity unrelated to any gauge symmetry, it is really more like an "invariantization" of the non-invariant stress tensor by applying more derivatives to it. Indeed, we can draw the following parallel with Maxwell theory. One may define a 4-index tensor there [4] which is essentially of the form

$$
\tau_{\mu \nu \alpha \beta} \sim \partial_{\alpha} F_{\mu \lambda} \partial_{\beta} F_{\nu}^{\lambda}+\ldots
$$


and consequently is also conserved on Maxwell shell, as is its more transparent trace

$$
\tau_{\mu \nu} \sim \partial_{\alpha} F_{\mu \lambda} \partial^{\alpha} F_{\nu}^{\lambda}-\frac{1}{4} \eta_{\mu \nu}\left(\partial_{\alpha} F_{\beta \gamma}\right)^{2}
$$

But in fact this quantity (in flat space) is just the wave operator acting on the Maxwell tensor (on-shell, which is all that counts, $\square F_{\mu \nu}=0$ ),

$$
\tau_{\mu \nu}=\frac{1}{2} \square T_{\mu \nu}^{\max },
$$

So such tensors, while rather superfluous for lower $(s \leq 1)$ spins, are really needed for higher ones. Indeed, all higher than $s=1$ gauge fields, already at the tree level, have non-gauge invariant stresstensors [6] and would require their own BR extensions, with more and more derivatives to emulate BR.

In summary, we have reviewed how stress tensors are surprisingly powerful for point and string - but not higher brane - systems: localized but otherwise arbitrary, their conservation suffices to specify all the dynamics. For lower spin fields, their actions specify the stress tensors but not the converse, as illustrated by the CS example: It is ambiguous to state that conservation occurs on - and only on - shell, since there can be more than one "shell". For gravity theories, there is no independent stress tensor but only a "covariantized", BR, version necessarily involving higher derivatives, hence requiring somewhat weaker conditions than Ricci-flatness for (covariant) conservation. Similar considerations hold also in attempting to endow other high spin gauge fields with gauge invariant stress tensors. These are however rather different conditions than for the fully covariant - gravity case. For example, free massless spin $>1$ fields also have non-invariant stress tensors, but their conservation is as directly linked to the (invariant) field equations as for lower spins.

The moral is that, while the standard lore is always essentially correct, there is always some fine print to take into account.

This work was supported by the National Science Foundation under grant PHY99-73935.

\section{References}

[1] A. Tulczjiew, Acta Phys. Pol. 18 (1939) 393.

[2] C. Aragone and S. Deser, Nucl. Phys. B92 (1975) 327.

[3] M. Gürses and F. Gürsey. Phys. Rev. D11 (1975) 967.

[4] M. Chevreton, Nuovo Cim. 34 (1964) 90.

[5] S. Deser, Class. Quant. Grav. 20 (2003) L213.

[6] S. Deser and J. McCarthy, Class. Quant. Grav. 7 (1990) L119; S. Deser and A. Waldron, in preparation. 